area closer than $900 \mathrm{~nm}$, the beads were moved in the direction of mycoplasma transiently. The bead displacements were measured and plotted as the relative position to the mycoplasma. The results showed that bead located in front of $M$ mobile moved toward M. mobile, and one located in the back of M.mobile moved away from $M$. mobile. These observations suggest that a foot of $M$. mobile catches and pulls sialic acid at front, and releases it when it is pulled forward at the tail side. We focused on the maximal displacement of bead in a passage, which depended on the position that $M$. mobile went along. When the distance between $M$. mobile and beads or the angle of the bead position to the moving direction was extrapolated to 0 , the displacement reached $85 \mathrm{~nm}$. The spring constant of the RBC membrane was estimated by optical tweezers, and the displacement of $85 \mathrm{~nm}$ was calculated to be $0.45 \mathrm{pN}, 1 / 50$ of the stall force of $M$. mobile.

\section{P-173 細胞集団の協同的振る舞いへの相転移}

\section{A phase transition to collective behavior in eukaryotic cell populations}

Thomas Gregor(1), Koichi Fujimoto(2) and Satoshi Sawai(1,2). (1: Graduate School of Arts and Sciences, University of Tokyo; 2: ERATO Complex Systems Biology Project, JST)

Collective dynamics are widely observed during multicellular development and result from communication among individual cells via signaling molecules. However, little is known experimentally how the highly nonlinear spatio-temporal dynamics at the single-cell level can give rise to coherent dynamics at the population level. The prototypical example is cell-cell signaling of social amoebae Dictyostelium, where a few hundred thousand cells aggregate to form a fruiting body. cAMP is synthesized and secreted periodically and serves as a cue that directs chemotaxis of individual cells. Here we use a FRET-based sensor protein, combined with live-imaging, to monitor cytosolic cAMP levels in developing Dictyostelium cells. Timelapse recordings during the first 10 hours of development reveal the very onset of periodic, spike-like signaling and sequential changes in the frequency at single cell resolution. The input-output relation for wild-type and mutant strains, obtained from single cells in isolation, indicates that the intracellular cAMP dynamics is governed by a PI3kinase-TORC2-actin dependent feedback loop. The cAMP oscillations in populations of cells under perfusion reveal a sharp transition between a decoupled state and collective behavior for a range of cell densities and dilution rates. These observations together with a mathematical model that we have constructed suggest that the intact population is able to drive itself to this transition spontaneously during development.

\section{P-174 ベん毛モーターコンポーネントのFCS 解析の実現に向けて}

Studies of interactions between flagellar rotor proteins bf Fluorescence Correlation Spectroscopy

Ryo Ogawa (1), Rei Yoshizumi (1), Sreiji Kojima (1), Michio Homma (1).(I:Division of Biological Science, Graduate school of Science Nagoya University)

Motile bacteria swim by means of flagella. At the base of each flagellum, there is a rotary motor powered by the electrochemical gradient of specific ion across the membrane. The motor consists of rotor and stator, and torque is generated by their interaction coupling with the ion flux through the stator. However, biochemical/biophysical analysis of the rotor-stator interaction has not proceeded, presumably because of their transient and weak interaction. Fluorescence Correlation Spectroscopy (FCS) has been used to detect weak protein-protein interaction in single molecule level, so we tried to detect rotor-stator interaction by this powerful method. FCS analysis of flagellar proteins has not yet established so this project also aims to establish FCS analysis to detect interactions between flagellar proteins. Rotor protein FliG binds to FliF ring in the flagellar basal body. So we chose FliG-FliF interaction for initial FCS measurement. Prior to examine FliF-FliG interaction, we carried out FCS measurement for FliG itself, and found that FliG self-associates with each other. FliG-FliG association in solution suggests that the some FliG molecules may form cluster. To elucidate FliG self-association more clearly, we will also try another methods called FIDA (Fluorescence Intensity Distribution Analysis) and FIDA-PO (FIDA-Polarization) method. FliF preparation is now ongoing, and further results including FliF-FliG interaction analyzed by FCS assay will be discussed in the poster.

\section{P-175 MARCKS の細胞膜近傍で 1 分子の挙動の研究}

Single fluorescent molecule tracking of MARCKS on the cytoplasmic surface of the cell membrane

Kokoro Iwasawal, Eriko Kajikawal, Ikuko Honda-Koyamal, Rinshi Kasail, Ken Ritchie2, Yoshihiro Miwa3, Akihiro Kusumil

The localization and the membrane microdomain association of MARCKS (Myristoylated Alanine-Rich C Kinase Substrate), which may associate laterally with the actin filament, and is known to bind to the cell membrane through a myristoyl chain as well as a basic effector domain that interacts with acidic PIP2, is drawing extensive attention. Furthermore, due to its possible lateral binding to actin filament, it might be involved in the partitioning of the plasma membrane based on the membrane skeleton meshwork. Here, we had MARCKS-GFP expressed in Hela cells, and carried out single-molecule tracking of MARCKS on the cell membrane, examining its recruitment to the cell membrane and its dynamics on the cytoplasmic surface of the cell membrane. Very low levels of MARCKS-GFP expression, circumventing effects of perturbing the cell and GFP-induced dimerization, were achieved by using tetracyclin-type EBV vector, containing the cDNA of MARCKS-GFP in tandem. Surprisingly, MARCKS-GFP rapidly moves back and forth between the cytoplasm and the cell membrane, residing on the cell membrane for less than a second on average for each recruitment event. Virtually all of the MARCKS-GFP molecules that stayed on the cell membrane longer than $100 \mathrm{~ms}$ undergo random diffusion with no indication of immobilization, with a median diffusion coefficient of 0.28 and $0.31 \mu^{2} / \mathrm{s}$ for GFP and monomeric GFP (A206K), respectively. It is concluded that the long-term binding of MARCKS to the actin membrane skeleton or membrane microdomains is unlikely.

\section{P-176 In vitro reconstitution of regulated $S$. pombe microtubule dynamics: the synergy of $S$. pombe EBI family member Mal3 and kinesin-7, Tea2.}

In vitro reconstitution of regulated $\mathrm{S}$. pombe microtubule dynamics: the synergy of S. pombe EB1 family member Mal3 and kinesin-7, Tea2.

Miho Katsuki(1) and Robert A. Cross(1) (1:Molecular Motors Group, Marie Curie Reaerch Institute)

Plus-end-tracking proteins (+Tips) form a complex at the plus end of microtubules (MT) and regulate MT dynamics. In S. pombe cells the +Tip EB1 homologue Mal3 interacts with Tea2, a kinesin-7 family member. In the absence of either protein, only short MTs are formed. Since it is difficult to determine the precise molecular function of each protein from cellular studies we established an in vitro reconstituted system utilising only proteins from $S$. pombe, which will permit us to compare directly in vitro dynamics with those in live S. pombe cells. Using our in vitro reconstituted system consisting of +TIPs expressed in $\mathrm{E}$. coli and purified single isoform S. pombe tubulin, we have found using a combination dark-field and fluorescence microscopy that Mal3 suppresses MT shrinkage rates without any enhancement of MT growth, and affects both MT catastrophe and rescue frequencies. These data indicate that Mal3 affects the MT lattice, as well as at the MT end. Mal3 promotes MT polymerisation in a light scattering assay. In addition to MT end binding, we found that in a MT pelleting assay Mal3 binds stoichiometrically to the MT lattice.Tea2 also binds to the MT lattice stoichiometrically, even when the MT lattice is saturated with Mal3. We have also found that although Tea2 has no effect by itself, it exhibits a powerful synergy with Mal3 to promote MT formation. This shows that Tea 2 may accelerate and potentiate the action of Mal3. We are now using Qdot fusion proteins to track the spatio-temporal trajectories of +TIPs on dynamic MTs in vitro.

\section{P-177 光ピンセット細胞触診システムによる細胞接着の力学的機構への} カ学的アプローチ

Mechanical approach to cell adhesion mechanism by using cell palpation system with optical tweezers

Hideaki MIYOSHI(1), Tadao SUGIURA(1) and Kotaro MINATO(I)(1:Department of Bioinformatics and Genomics, Nara Institute of Science and Technology)

Cell adhesion is affected by interactions of extracellular matrix (ECM) and cytoskeleton. Especially, force provided by cytoskeleton over focal adhesions (FA) plays important roles in cell morphology, motility, growth, and differentiation. Such mechanical functions in a cell are gradually revealed by various approaches based on molecular and structure biological technique. To connect these molecular mechanism and mechanical functions, a mechanical approach, in which we investigate mechanical forces concerning to specific functions at a single focal adhesion, is necessary. In this study, we propose a technique for force measurement at a single FA-ECM complex. We have developed a measurement system of mechanical characteristic in local of a cell by using a cell palpation system, which is proposed recently. In this system a micron-sized particle is manipulated by optical tweezers and is adhered on a cell to be measured, and then force is induced on cell membrane. We use a particle whose surface is modified by carboxyl group, to achieve nonspecific binding to cell membrane. We can investigate mechanical properties of various kinds of structures, which include cell membrane, membrane protein and cytoskeleton, through measurements of force on the particle attached on a small area of the cell by analysis based on mechanical model of the particle. We report measuremen results taken by ECM coated particles and a relationship between mechanical properties and mechanisms of cell adhesion at molecular level.

\section{$3 \mathrm{P}-178$ 細胞外刺激に対するシグナル伝達ネットワークの応答を記述する ための理論的研究}

Computer simulation and theoretical formulation of stimulus/response relationships of intracellular signaling network cascades

Tatsunori Nishimura(1), Thomas Maiwald(2), Jens Timmer(2) and Masaru Tateno(2) (1: Center for Computational Sciences, University of Tsukuba; 2: Freiburg Center for Dato Analysis and Modeling, University of Freiburg, Germany)

A typical mechanism of intracellular signal transduction is sequential phosphorylations of inactive proteins by activated factors, thus leading to complicated reaction cascades. According to concentrations of extracellular signaling molecules (intensity of stimulus), ratios of active proteins at the steady state show characteristic behaviors (the stimulus/response relationship), such as sigmoidal responses and noise-reduction effects (the latter is characterized by thresholds in stimulus/response curves).

In the previous studies, the Hill equation, which was originated from a model to describe $\mathrm{O}_{2}$ absorption mechanisms by hemoglobin, was just formally applied to attempt to reproduce the behavior of signaling cascades; it was not consistent in ranges of both low and high stimulus, although the sigmoidal behavior was fitted. In this study, we propose a theoretical formulation to strictly describe cellular 